\title{
CONSISTENCE OF A GL(3,R) GAUGE FORMULATION FOR TOPOLOGICAL MASSIVE GRAVITY 1
}

\author{
Rolando Gaitan D. \\ Departamento de Física, Facultad de Ciencias y Tecnología, \\ Universidad de Carabobo, A.P. 129 Valencia 2001, Edo. Carabobo, Venezuela. \\ e-mail:rgaitan@uc.edu.ve
}

\begin{abstract}
We include a Chern-Simons term in a $G L(3, R)$ gauge formulation of gravity with a cosmological contribution in $2+1$ dimension and we explore consistence showing that excitations must be causal and standard topological massive gravity is recovered from this type of construction at the torsionless limit.
\end{abstract}

\section{Introduction}

It is well known that the introduction of a Chern-Simons lagrangian term (CS) in the Hilbert-Einstein formulation provides a theory which describes a massive excitation of a graviton in $2+1$ dimensions[1]. If a cosmological term is included, the cosmologically extended topological massive gravity (TMG $\lambda$ ) arises[2]. The aforementioned action is

$$
S=\frac{1}{\kappa^{2}} \int d^{3} x \sqrt{-g}(R+\lambda)+\frac{1}{\kappa^{2} \mu} S_{C S},
$$

where $\kappa^{2}$ is in lenght units (i.e., $\kappa^{2} \sim l$ ), $\mu \sim l^{-1}$ and $S_{C S}$ is the CS action. In a Riemannian space-time, the action (11) gives the field equation, $R^{\mu \nu}-\frac{g^{\mu \nu}}{2} R-\lambda g^{\mu \nu}+$ $\frac{1}{\mu} C^{\mu \nu}=0$ where $C^{\mu \nu}$ is the (traceless) Cotton tensor. The trace of the field equation

\footnotetext{
${ }^{1}$ Talk given at the Spanish Relativity Meeting 2007, Relativistic Astrophysics and Cosmology, Puerto de la Cruz, Tenerife, Spain
} 
gives a consistency condition on the trace of the Ricci tensor (this means, $R=-6 \lambda$ ). Starting with the field equation, it is possible to write down an hyperbolic-causal equation which describes a massive propagation for the Ricci tensor as follows

$$
\begin{array}{r}
\left(\nabla_{\mu} \nabla^{\mu}-\mu^{2}\right) R_{\mu \nu}-R^{\alpha \beta} R_{\alpha \beta} g_{\mu \nu}+3 R_{\mu}^{\alpha} R_{\alpha \nu}+\frac{\mu^{2}}{3} R g_{\mu \nu} \\
-\frac{3}{2} R R_{\mu \nu}+\frac{1}{2} R^{2} g_{\mu \nu}=0 .
\end{array}
$$

The next section is devoted to explore consistence of a $G L(3, R)$ gauge formulation $[3,4]$ for topological massive gravity with cosmological constant (GTMG $\lambda$ ), verifying the existence of causal propagation and the fact that standard TMG $\lambda$ can be recovered from GTMG $\lambda$ at the torsionless limit. Some remarks will be given in the conclusions.

\section{A GL(3,R) gauge formulation for topological mas- sive gravity with cosmological constant}

A brief review of the gauge formulation for (free) gravity with cosmological constant starts here[4]. Let $M$ be a $2+1$ dimensional manifold with a metric, $g_{\mu \nu}$ provided. A (principal) fiber bundle is constructed with $M$, a 1-form connection is given, $\left(A_{\lambda}\right)^{\mu}{ }_{\nu}$ which will be though non metric dependent. The connection transforms as $A_{\lambda}{ }^{\prime}=$ $U A_{\lambda} U^{-1}+U \partial_{\lambda} U^{-1}$ under $U \in G L(3, R)$. Torsion and curvature tensors are $T_{\lambda \nu}^{\mu}=$ $\left(A_{\lambda}\right)^{\mu}{ }_{\nu}-\left(A_{\nu}\right)_{\lambda}^{\mu}$ and $F_{\mu \nu} \equiv D_{\mu} A_{\nu}-D_{\nu} A_{\mu}+\left[A_{\mu}, A_{\nu}\right]$ (components of the Riemann tensor are $\left.R_{\alpha \mu \nu}^{\sigma} \equiv\left(F_{\nu \mu}\right)_{\alpha}^{\sigma}\right)$. The gauge invariant action is

$$
S_{o}=\kappa^{2} \int d^{3} x \sqrt{-g}\left(-\frac{1}{4} \operatorname{tr} F^{\alpha \beta} F_{\alpha \beta}+\lambda^{2}\right) .
$$

which reproduces the Hilbert-Einstein with cosmological constant field equations.

The lagrangian massive term to consider is the CS action

$$
S_{C S}=\frac{m \kappa^{2}}{2} \int d^{3} x \epsilon^{\mu \nu \lambda} \operatorname{tr}\left(A_{\mu} \partial_{\nu} A_{\lambda}+\frac{2}{3} A_{\mu} A_{\nu} A_{\lambda}\right)
$$


which is gauge variant because

$$
\delta_{U} S_{C S}=-\frac{m \kappa^{2}}{2} \int d^{3} x \epsilon^{\mu \nu \lambda} \operatorname{tr} \partial_{\nu}\left[A_{\mu} \partial_{\lambda} U U^{-1}\right]-4 \pi^{2} \kappa^{2} m W(U)
$$

where $W(U) \equiv \frac{1}{24 \pi^{2}} \int d^{3} x \epsilon^{\mu \nu \lambda} \operatorname{tr}\left(U^{-1} \partial_{\mu} U U^{-1} \partial_{\nu} U U^{-1} \partial_{\lambda} U\right)$ is the "winding number" of the gauge transformation $U$. So, the topological massive action is

$$
S=S_{o}+S_{C S}
$$

The torsionless limit of (6) can be explored by introducing nine constraints through the new action $S^{\prime}=S+\kappa^{2} \int d^{3} x \sqrt{-g} b_{\alpha \beta} \varepsilon^{\beta \lambda \sigma}\left(A_{\lambda}\right)_{\sigma}^{\alpha}$, where $b_{\alpha \beta}$ are lagrange multipliers. Variation on connection and metric gives rise to the following field equations

$$
\begin{gathered}
D_{\mu} R_{\sigma \lambda}-D_{\lambda} R_{\sigma \mu}-m \varepsilon^{\nu \rho}{ }_{\sigma}\left(g_{\lambda \nu} R_{\mu \rho}-g_{\mu \nu} R_{\lambda \rho}-\frac{2}{3} R g_{\lambda \nu} g_{\mu \rho}\right)=0 \\
R_{\sigma \mu} R^{\sigma}{ }_{\nu}-R R_{\mu \nu}+\frac{g_{\mu \nu}}{4} R^{2}-g_{\mu \nu} \lambda^{2}=0
\end{gathered}
$$

where the following consistency condition appears

$$
R=\text { constant }
$$

Due to the last condition on the Ricci scalar, we can test solutions of the type $R_{\mu \nu}=\frac{R}{3} g_{\mu \nu}$, by pluging them in (8), and this gives

$$
R= \pm 6|\lambda|
$$

verifying the existence of (Anti) de Sitter solutions.

A quick look on causal propagation of the theory can be performed writing a second order equation from (7), this means

$$
\begin{array}{r}
\left(\nabla_{\alpha} \nabla^{\alpha}-m^{2}\right) R_{\mu \nu}-R^{\alpha \beta} R_{\alpha \beta} g_{\mu \nu}+3 R_{\mu}^{\alpha} R_{\alpha \nu}+\frac{m^{2} R}{3} g_{\mu \nu} \\
-\frac{3 R}{2} R_{\mu \nu}+\frac{R^{2}}{2} g_{\mu \nu}=0 .
\end{array}
$$




\section{Conclusion}

Equation (11) describes a massive hyperbolic-causal propagation of graviton. So, GTMG $\lambda$ contains as a particular case the TMG $\lambda$ classical formulation (at the torsionless limit) if we take the mass value $m$ as the CS $(m=\mu)$ and the consistency condition (9) is fixed as (10).

Obviously, GTMG $\lambda$ is gauge variant under $G L(3, R)$ due to the presence of the CS term. However, by taking boundary conditions on the elements $U$, the term $\int d^{3} x \epsilon^{\mu \nu \lambda} \operatorname{tr} \partial_{\nu}\left[A_{\mu} \partial_{\lambda} U U^{-1}\right]$ in (5), goes to zero and the transformation rule now is $\delta_{U} S=-4 \pi^{2} \kappa^{2} m W(U)$. If we demand that the expectation value of a gauge invariant operator (i.e., $<\mathcal{O}>\equiv Z^{-1} \int \mathcal{D} A \mathcal{O}(A) e^{i S}$ with the gauge invariant measure $\mathcal{D} A$ and the normalization constant $Z$ ) must be gauge invariant too, it is required that $-4 \pi^{2} \kappa^{2} m W(U)$ be an integral multiple of $2 \pi$ and a quantization condition on the parameter $\kappa^{2} m$ must arises. This fact occurs, at least, by performing a restriction on the covariance of the theory, this means, taking a compact subgroup of $G L(3, R)$ (i.e., $S O(3))$.

A first step to explore a canonical quantization program for this theory and a study of propagation of spin degree of freedom, between other things, could be the linearization of the metric and connection. There, the perturbative analysis must depends non trivially on which kind of non perturbed space-time we start (i.e., torsionless or not). This will be studied elsewhere.

\section{Acknowlegdment}

Author wish to thanks M. Valera for technical support. This work is supported by Grants FONACIT G-2001000712 and CDCH-UC 1102-06.

\section{References}

[1] Deser, S., Jackiw, R., Templeton, S., 1982, Ann. of Phys. 140, 372 
[2] Deser, S., 1984, "Cosmological Topological Supergravity" in Quantum Theory of Gravity, Ed. S. M. Christensen, Adam Hilger, London

[3] Mansouri, F., Chang, L.N., 1976, Phys. Rev. D13, 12, 3192

[4] Gaitan, R., 2003, Mod. Phys. Lett. A, 18, 1753 